\title{
Extension of Matched Asymptotic Method to Fractional Boundary Layers Problems
}

\author{
Abdon Atangana ${ }^{1}$ and Emile Franc Doungmo Goufo ${ }^{2}$ \\ ${ }^{1}$ Institute for Groundwater Studies, Faculty of Natural and Agricultural Sciences, University of the Free State, \\ Bloemfontein 9300, South Africa \\ ${ }^{2}$ Department of Mathematical Sciences, University of South Africa, Florida Sciences Campus, Florida 0003, South Africa
}

Correspondence should be addressed to Emile Franc Doungmo Goufo; dgoufef@unisa.ac.za

Received 16 June 2014; Revised 24 July 2014; Accepted 24 July 2014; Published 20 November 2014

Academic Editor: Hossein Jafari

Copyright (C) 2014 A. Atangana and E. F. Doungmo Goufo. This is an open access article distributed under the Creative Commons Attribution License, which permits unrestricted use, distribution, and reproduction in any medium, provided the original work is properly cited.

\begin{abstract}
We were concerned with the description of the boundary layers problems within the scope of fractional calculus. However, we will note that one of the main methods used to solve these problems is the matched asymptotic method. We should mention that this was not achievable via the existing fractional derivative definitions, because they do not obey the chain rule. In order to accommodate the matched asymptotic method to the scope of fractional derivative, we proposed a relatively new derivative called the beta-derivative. We presented some useful information for this operator. With the reward of this operator, we presented the idea of matched asymptotic method in finding solutions of the fractional boundary layers problems. The method was illustrated with an example.
\end{abstract}

\section{Introduction}

In a large class of singular perturbed problems, the domain may be divided into two or more subdomains. In one of these, often the largest, the solution is accurately approximated by an asymptotic series found by treating as a regular perturbation. The other subdomains consist of one or more small areas in which that approximation is inaccurate, generally because the perturbation terms in the problem are not negligible there. These areas are referred to as transition layers and as boundary or interior layers depending on whether they occur at the domain boundary or inside the domain [1-6]. An approximation in the form of an asymptotic series is obtained in transition layers by treating that part of the domain as a separate perturbation problem. This approximation is called the "inner solution," and the other is the "outer solution" named for their relationship to transition layers. The outer and inner solutions are then combined through a process called "matching" in such a way that an approximate solution for the whole domain is obtained [1-6]. Therefore, the matched asymptotic method is a common approach to finding an accurate approximation to solution to an equation in particular when solving perturbed differential equation with conventional order derivative. This class of differential equations is used to describe real world problems; for instance, in physic and fluid mechanics, a boundary layer is the layer of fluid in the immediate neighbourhood of a bouncing surface where the consequences of viscidness are noteworthy. In the earth's atmosphere, the environmental borderline layer is the air layer neighbouring the earth affected by diurnal heat, moisture, or momentum transfer to or from the surface. On an aircraft wing, the boundary layer is the part of the flow close to the wing, where viscous forces distort the surrounding nonviscous flow.

In the recent decades, attention has been paid by several scholars to modeling the real world problems with the concept of fractional order derivatives. It was revealed by many proofs and results that the modelling of these real world problems with the concept of fractional order derivatives gives better prediction rather than using conventional derivative, which is regarded as integer order derivatives.

It is perhaps important to mention that the matched asymptotic method has never been used to solve any kind of fractional differential equations because of the nature 
and properties of the fractional derivative. In particular, the most commonly used fractional derivative (Caputo fractional derivative) for modelling the real world problem does not obey the chain rule which is one of the key elements of the matched asymptotic method. Recently, the so-called conformable fractional derivative was proposed. This fractional derivative is theoretically very easier to handle and also obeys some conventional properties that cannot be satisfied by the existing fractional derivatives, for instance, the chain rule. However this fractional derivative has a very big weakness, which is the fractional derivative of any differentiable function at the point zero and this does not satisfy any physical problem or cannot for the moment have any physical interpretation. A modified version was proposed in order to extend the limitation of the conformable derivative; however this derivative depends on the interval on which the function is being differentiated which is also a true problem for some physical problem.

The aim of this work is to further extend the so-called matched asymptotic method in the scope of the fractional differential equation on the one hand. On the other hand, we will propose a suitable derivative using fractional order, that is easier and respects the chain rule. This version of fractional derivative will be used to further describe the boundary layers problems within the folder of fractional calculus. We will start with some existing definitions of fractional derivatives in the coming section.

\section{Some Definitions of Fractional Derivatives}

There exist a quite few definitions of fractional derivatives in the literature; we will present the definition of a few [7-12].

(1) The Riemann-Liouville fractional derivative of a function says $f$ is defined as

$$
\begin{array}{r}
D_{x}^{\alpha}(f(x))=\frac{1}{\Gamma(n-\alpha)}\left(\frac{d}{d x}\right)^{n} \int_{0}^{x}(x-t)^{n-\alpha-1} f(t) d t \\
n-1<\alpha \leq n .
\end{array}
$$

(2) The Caputo fractional derivative of a differentiable function says $f$ is defined as

$$
\begin{array}{r}
D_{x}^{\alpha}(f(x))=\frac{1}{\Gamma(n-\alpha)} \int_{0}^{x}(x-t)^{n-\alpha-1}\left(\frac{d}{d t}\right)^{n} f(t) d t \\
n-1<\alpha \leq n .
\end{array}
$$

(3) The modified Liouville fractional derivative of a function says $f$ is defined as

$$
\begin{aligned}
D_{x}^{\alpha}(f(x))= & \frac{1}{\Gamma(n-\alpha)}\left(\frac{d}{d x}\right)^{n} \\
& \times \int_{0}^{x}(x-t)^{n-\alpha-1}(f(t)-f(0)) d t, \\
& n-1<\alpha \leq n .
\end{aligned}
$$

(4) The local fractional derivative of a function says $f$ is defined as

$$
L_{x}^{\alpha}(f(x))=\lim _{x \rightarrow x_{0}} \frac{\Gamma(1+\alpha)\left(f(x)-f\left(x_{0}\right)\right)}{\left(x-x_{0}\right)^{\alpha}} .
$$

(5) The conformable fractional derivative of a function says $f$ is given as

$$
T_{\alpha}(f(x))=\lim _{\varepsilon \rightarrow 0} \frac{f\left(x+\varepsilon x^{1-\alpha}\right)-f(x)}{\varepsilon} .
$$

(6) The modified conformable fractional derivative of a given function $f$ defined in the interval $(a, b)$ is given as

$$
T_{\alpha}(f(x))=\lim _{\varepsilon \rightarrow 0} \frac{f\left(x+\varepsilon(x-a)^{1-\alpha}\right)-f(x)}{\varepsilon} .
$$

The last two seem to satisfy some common properties of the standard concept of derivative, but as we said before they have some limitations that will not allow them to be used in modelling real world problems. We will therefore propose a suitable fractional derivative that will allow us to escape the lack of these fractional derivatives.

Definition 1. Let $f$ be a function, such that $f:[a, \infty) \rightarrow \mathbb{R}$. Then, the beta derivative of a function $f$ is defined as

$$
{ }_{0}^{A} D_{x}^{\beta}(f(x))=\lim _{\varepsilon \rightarrow 0} \frac{f\left(x+\varepsilon(x+1 / \Gamma(\beta))^{1-\beta}\right)-f(x)}{\varepsilon},
$$

for all $x \geq a, \beta \in(0,1]$. Then if the limit of the above exists, $f$ is said to be $\beta$-differentiable.

One can remark that the above definition does not depend on the interval on which the function is defined as in the case of Definition 6 . If the function is differentiable, our definition at a point zero is different to zero, which was not the case for the 5 definitions.

Theorem 2. Assuming that a given function says $f:[a, \infty)$ $\rightarrow \mathbb{R}$ is $\beta$-differentiable at the points says $x_{0} \geq a, \beta \in(0,1]$, then $f$ is also continuous at $x_{0}$.

Proof. Assuming that $f$ is $\beta$-differentiable, then

$$
\begin{aligned}
{ }_{0}^{A} D_{x}^{\beta}\left(f\left(x_{0}\right)\right) \\
=\lim _{\varepsilon \rightarrow 0} \frac{f\left(x_{0}+\varepsilon\left(x_{0}+1 / \Gamma(\beta)\right)^{1-\beta}\right)-f\left(x_{0}\right)}{\varepsilon}
\end{aligned}
$$

exists. Therefore,

$$
\begin{aligned}
& \lim _{\varepsilon \rightarrow 0}\left(f\left(x_{0}+\varepsilon\left(x_{0}+\frac{1}{\Gamma(\beta)}\right)^{1-\beta}\right)-f\left(x_{0}\right)\right) \\
& =\lim _{\varepsilon \rightarrow 0} \frac{f\left(x_{0}+\varepsilon\left(x_{0}+1 / \Gamma(\beta)\right)^{1-\beta}\right)-f\left(x_{0}\right)}{\varepsilon} \varepsilon \\
& ={ }^{A} D_{x}^{\beta}\left(f\left(x_{0}\right)\right) \cdot 0=0,
\end{aligned}
$$


thus giving

$$
\begin{aligned}
& \lim _{\varepsilon \rightarrow 0} f\left(x_{0}+\varepsilon\left(x_{0}+\frac{1}{\Gamma(\beta)}\right)^{1-\beta}\right) \\
& \quad=\lim _{\varepsilon \rightarrow 0}\left(f\left(x_{0}+\varepsilon\left(x_{0}+\frac{1}{\Gamma(\beta)}\right)^{1-\beta}\right)-f\left(x_{0}\right)\right)+f\left(x_{0}\right) \\
& =0+f\left(x_{0}\right) .
\end{aligned}
$$

However, taking $x=x_{0}+\varepsilon\left(x_{0}+1 / \Gamma(\beta)\right)^{1-\beta}$ so that $\varepsilon=(x-$ $\left.x_{0}\right) /\left(x_{0}+1 / \Gamma(\beta)\right)^{1-\beta}$, thus

$$
\lim _{\left(x-x_{0}\right) /\left(x_{0}+1 / \Gamma(\beta)\right)^{1-\beta} \rightarrow 0} f(x)=f\left(x_{0}\right) ;
$$

since $\left(x_{0}+1 / \Gamma(\beta)\right)^{1-\beta} \neq 0$, then the above can be rewritten as

$$
\lim _{x \rightarrow x_{0} \rightarrow 0} f(x)=f\left(x_{0}\right) \quad \text { or } \quad \lim _{x \rightarrow x_{0}} f(x)=f\left(x_{0}\right),
$$

which completes the proof.

Theorem 3. Assuming that $f$ is $\beta$-differentiable on an open interval $(a, b)$, then

(1) if ${ }_{0}^{A} D_{x}^{\beta}(f(x))<0$ for all $x \in(a, b)$, then $f$ is decreasing there;

(2) if ${ }_{0}^{A} D_{x}^{\beta}(f(x))>0$ for all $x \in(a, b)$, then $f$ is increasing there;

(3) if ${ }_{0}^{A} D_{x}^{\beta}(f(x))=0$ for all $x \in(a, b)$, then $f$ is constant there.

Proof. Assume that $f$ is $\beta$-differentiable on an open interval $(a, b)$ and ${ }_{0}^{A} D_{x}^{\beta}(f(x))>0$; then, consider $x_{1}>x_{2}$

$$
\frac{f\left(x_{1}+\varepsilon\left(x_{2}+1 / \Gamma(\beta)\right)^{1-\beta}\right)-f\left(x_{2}\right)}{\varepsilon}>0 \text {. }
$$

Then,

$$
f\left(x_{1}+\varepsilon\left(x_{2}+\frac{1}{\Gamma(\beta)}\right)^{1-\beta}\right)-f\left(x_{2}\right)>0 .
$$

Now, taking the limit on both sides, we have that

$$
\begin{aligned}
& \lim _{\varepsilon \rightarrow 0} f\left(x_{1}+\varepsilon\left(x_{2}+\frac{1}{\Gamma(\beta)}\right)^{1-\beta}\right)-f\left(x_{2}\right) \\
& =f\left(x_{1}\right)-f\left(x_{2}\right)>0 .
\end{aligned}
$$

This completes the proof. Note that, by using a similar approach, we can also prove 2 and 3 .

Theorem 4. Assuming that $g \neq 0$ and $f$ are two functions $\beta$ differentiable with $\beta \in(0,1]$, then the following relations can be satisfied:
(1) ${ }_{0}^{A} D_{x}^{\alpha}(a f(x)+b g(x))=a_{0}^{A} D_{x}^{\alpha}(f(x))+b_{0}^{A} D_{x}^{\alpha}(f(x))$ for all $a$ and $b$ real number;
(2) ${ }_{0}^{A} D_{x}^{\alpha}(c)=0$ for $c$ any given constant;
(3) ${ }_{0}^{A} D_{x}^{\alpha}(f(x) g(x))=g(x){ }_{0}^{A} D_{x}^{\alpha}(f(x))+f(x){ }_{0}^{A} D_{x}^{\alpha}(g(x))$;
(4) ${ }_{0}^{A} D_{x}^{\alpha}(f(x) / g(x))=\left(g(x){ }_{0}^{A} D_{x}^{\alpha}(f(x))-f(x){ }_{0}^{A} D_{x}^{\alpha}(g(x))\right) /$ $g^{2}(x)$.

The proofs of the above relations are the same as the one in [12].

Theorem 5. Let $f:[a, \infty) \rightarrow \mathbb{R}$ be a function such that $f$ is differentiable and also $\alpha$-differentiable. Let $g$ be a function defined in the range of $f$ and also differentiable; then, one has the following rule:

$$
{ }_{0}^{A} D_{x}^{\beta}(g \circ f(x))=\left(x+\frac{1}{\Gamma(\beta)}\right)^{1-\beta} f^{\prime}(x) g^{\prime}(f(x)) .
$$

Proof. We have from the definition that

$$
\lim _{\varepsilon \rightarrow 0} \frac{\operatorname{gof}\left(x+\varepsilon(x+1 / \Gamma(\beta))^{1-\beta}\right)-\operatorname{gof}(x)}{\varepsilon} .
$$

Let $h=\varepsilon(x+1 / \Gamma(\beta))^{1-\beta}$ such that

$$
\begin{aligned}
& \lim _{\varepsilon \rightarrow 0} \frac{\operatorname{gof}\left(x+\varepsilon(x+1 / \Gamma(\beta))^{1-\beta}\right)-\operatorname{gof}(x)}{\varepsilon} \\
& \quad=\left(x+\frac{1}{\Gamma(\beta)}\right)^{1-\beta} \lim _{h \rightarrow 0} \frac{\operatorname{gof}(x+h)-\operatorname{gof}(x)}{h} .
\end{aligned}
$$

According to chain rule, we have that

$$
\lim _{h \rightarrow 0} \frac{g o f(x+h)-g \circ f(x)}{h}=f^{\prime}(x) g^{\prime}(f(x)) .
$$

Therefore, we have

$$
{ }^{A} D_{x}^{\beta}(g \circ f(x))=\left(x+\frac{1}{\Gamma(\beta)}\right)^{1-\beta} f^{\prime}(x) g^{\prime}(f(x)) .
$$

The above rule is referred to as Atangana beta-rule. We will note that if $\beta=1$, we recover the Chain rule. We will present new derivative for some special functions.

(1) The beta-derivative of the Mittag-Leffler is given as

$$
{ }_{0}^{A} D_{x}^{\beta}\left[E_{\alpha}(x)\right]=\left(x+\frac{1}{\Gamma(\beta)}\right)^{1-\beta} \sum_{n=1}^{\infty} \frac{n x^{n-1}}{\Gamma(\alpha n+1)} .
$$

(2) The beta-derivative of Bessel function of first kind is given as

$$
\begin{aligned}
{ }_{0}^{A} D_{x}^{\beta}\left[J_{v}(x)\right]= & \frac{1}{2}\left(x+\frac{1}{\Gamma(\beta)}\right)^{1-\beta} \\
& \times \sum_{n=1}^{\infty} \frac{(-1)^{n}}{(n-1) !}(2 n+v) \frac{(x / 2)^{2 n+v-1}}{n ! \Gamma(v+n+1)} .
\end{aligned}
$$


(3) The beta-derivative of Error function is given as

$$
{ }_{0}^{A} D_{x}^{\beta}[\operatorname{Erf}(x)]=\left(x+\frac{1}{\Gamma(\beta)}\right)^{1-\beta} \frac{2}{\sqrt{\pi}} e^{-x^{2}} .
$$

We can carry on and find all the fractional derivatives of the special functions; however, we will notice that if $\beta=1$, we obtain without any problem their derivatives with the standard derivative. We will now look at the inverse operator of the proposed fractional derivative.

Definition 6. Let $f:[a, \infty) \rightarrow \mathbb{R}$ be given function; then one proposes that Atangana's beta-integral of $f$ is

$$
{ }_{a}^{A} I_{x}^{\beta}(f(x))=\int_{a}^{x}\left(t+\frac{1}{\Gamma(\beta)}\right)^{\beta-1} f(t) d t .
$$

The above operator is the inverse operator of the proposed fractional derivative. One will present to underpin this statement by the following theorem.

Theorem 7. ${ }_{0}^{A} D_{x}^{\alpha}\left[{ }_{0}^{A} I_{x}^{\alpha} f(x)\right]=f(x)$ for all $x \geq a$ with $f$ being a given continuous and differentiable function.

Proof. Let $f$ be a continuous function; then, by definition, if we let ${ }_{0}^{A} I_{x}^{\alpha} f(x)=F(x)$, we have

$$
\begin{aligned}
{ }_{0}^{A} D_{x}^{\alpha}\left[{ }_{0}^{A} I_{x}^{\alpha} f(x)\right]= & \lim _{\varepsilon \rightarrow 0} \frac{F\left(x+\varepsilon(x+1 / \Gamma(\beta))^{1-\beta}\right)-F(x)}{\varepsilon} \\
= & \left(x+\frac{1}{\Gamma(\beta)}\right)^{1-\beta} \frac{d F(x)}{d x} \\
= & \left(x+\frac{1}{\Gamma(\beta)}\right)^{1-\beta} \frac{d}{d x} \\
& \times\left\{\int_{a}^{x}\left(t+\frac{1}{\Gamma(\beta)}\right)^{\beta-1} f(t) d t\right\} \\
= & \left(x+\frac{1}{\Gamma(\beta)}\right)^{1-\beta}\left(x+\frac{1}{\Gamma(\beta)}\right)^{\beta-1} f(x) \\
= & f(x) .
\end{aligned}
$$

This completes the proof.

Theorem 8. ${ }_{a}^{A} I_{x}^{\alpha}\left[{ }_{0}^{A} D_{x}^{\alpha} f(x)\right]=f(x)-f(a)$ for all $x \geq a$ with $f$ being a given continuous and differentiable function.

Proof. Since $f$ is continuous and differentiable, by definition, we have that

$$
\begin{aligned}
{ }_{a}^{A} I_{x}^{\alpha}\left[{ }_{0}^{A} D_{x}^{\alpha} f(x)\right] & =\int_{a}^{x}\left(t+\frac{1}{\Gamma(\beta)}\right)^{\beta-1}{ }^{A} D_{t}^{\alpha} f(t) d t \\
& =\int_{a}^{x}\left(t+\frac{1}{\Gamma(\beta)}\right)^{\beta-1}\left(t+\frac{1}{\Gamma(\beta)}\right)^{1-\beta}
\end{aligned}
$$

$$
\begin{gathered}
\times \frac{d f(x)}{d x} d t \\
=\int_{a}^{x} \frac{d f(x)}{d x} d t=f(x)-f(a) .
\end{gathered}
$$

This completes the proof.

We will present beta-integral of some simple functions.

(1) For $f(x)=c$ as given constant, we have

$$
\begin{aligned}
{ }_{0}^{A} I_{x}^{\alpha}[c] & =\int_{0}^{x}\left(t+\frac{1}{\Gamma(\beta)}\right)^{\beta-1} c d t \\
& =\frac{c \Gamma[\alpha]^{-\alpha}\left(-1+(1+x \Gamma[\alpha])^{\alpha}\right)}{\alpha} .
\end{aligned}
$$

(2) For $f(x)=c x^{n}$ for any given natural number $n$ and real number $a$, we have

$$
\begin{aligned}
& \int_{0}^{x}\left(t+\frac{1}{\Gamma(\beta)}\right)^{\beta-1} c t^{n} d t \\
& \quad=-c(-x)^{-n} x^{n} \operatorname{Beta}[-x \Gamma[\alpha], 1+n, \alpha] \Gamma[\alpha]^{-n-\alpha}
\end{aligned}
$$

(3) For $f(x)=\cos (x)$, we have the following:

$$
\begin{aligned}
\int_{0}^{x}(t & \left.+\frac{1}{\Gamma(\beta)}\right)^{\beta-1} \cos [t] d t \\
= & -\frac{1}{2} e^{-(1 / 2) i \pi \alpha} \\
\times & \left(-e^{i \pi \alpha} \Gamma\left[\alpha,-\frac{i}{\Gamma[\alpha]}\right]\right. \\
& \times\left(\cos \left[\frac{1}{\Gamma[\alpha]}\right]-i \sin \left[\frac{1}{\Gamma[\alpha]}\right]\right) \\
& \left.-\Gamma\left[\alpha, \frac{i}{\Gamma[\alpha]}\right]\left(\cos \left[\frac{1}{\Gamma[\alpha]}\right]+i \sin \left[\frac{1}{\Gamma[\alpha]}\right]\right)\right) \\
& \frac{1}{2}(1+x \Gamma[\alpha])^{\alpha}\left((1+x \Gamma[\alpha])^{2}\right)^{-\alpha} \\
\times & \left(\Gamma[\alpha]^{\alpha} \Gamma\left[\alpha,-\frac{i(1+x \Gamma[\alpha])}{\Gamma[\alpha]}\right]\right. \\
& \times\left(\begin{array}{c}
\alpha(\operatorname{Conjugate}[\log [i(1+x \Gamma[\alpha])]]-\log [\Gamma[\alpha]]) \\
\Gamma[\alpha] \\
\left(\frac{i(1+x \Gamma[\alpha])}{\Gamma[\alpha]}\right)^{\alpha}
\end{array}\right)
\end{aligned}
$$




$$
\begin{aligned}
& \times\left(\cos \left[\frac{1}{\Gamma[\alpha]}\right]-i \sin \left[\frac{1}{\Gamma[\alpha]}\right]\right) \\
& +(-i(1+x \Gamma[\alpha]))^{\alpha} \Gamma \\
& \times\left[\alpha, \frac{i(1+x \Gamma[\alpha])}{\Gamma[\alpha]}\right] \\
& \left.\times\left(\cos \left[\frac{1}{\Gamma[\alpha]}\right]+i \sin \left[\frac{1}{\Gamma[\alpha]}\right]\right)\right) .
\end{aligned}
$$

(4) For $f(x)=\exp [t]$, we have the following:

$$
\begin{aligned}
\int_{0}^{x}\left(t+\frac{1}{\Gamma(\beta)}\right)^{\beta-1} & \cos [t] d t \\
=e^{-(1 / \Gamma[\alpha])}(( & -1-x \Gamma[\alpha])^{-\alpha}(1+x \Gamma[\alpha])^{\alpha} \\
& \times\left(\Gamma[\alpha]-\Gamma\left[\alpha,-x-\frac{1}{\Gamma[\alpha]}\right]\right) \\
& \left.+(-1)^{-\alpha}\left(-\Gamma[\alpha]+\Gamma\left[\alpha,-\frac{1}{\Gamma[\alpha]}\right]\right)\right) .
\end{aligned}
$$

Other properties of the proposed operator will be investigated in the next work. However, we will now present the application of this operator in the scope of fractional boundary layers problems.

\section{A Matched Asymptotic Method for Fractional Boundary Layer Problems}

In this section, we will present the discussion that underpins the extension of the so-called matched asymptotic method in the scope of fractional boundary layer problem. The fractional derivative used here will be the beta-derivative because it provides us with useful rule called the new chain rule that cannot be obtained by the existing fractional derivative operator. We will then present the general methodology. Many singular perturbed differential equations have solutions that change rapidly in a narrow region. This may occur in an initial layer where there is a rapid adjustment of initial differential conditions to a quasisteady state, in a boundary layer where the solution away from the boundary adjusts to a boundary condition, or in an interior layer such as a propagation wave front. This class of problems will be further investigated in the scope of fractional calculus by using the fractional matched asymptotic method.

Consider the following fractional singular perturbation problem:

$$
P^{\alpha}(u(x, \varepsilon))=0, \quad u(a)=A, \quad u(b)=B,
$$

where $\alpha \geq 1$ is the fractional order derivative. To solve the above equation, we assume that

$$
u(x, \varepsilon)=\sum_{n=0}^{\infty} \varepsilon^{n} u_{n}(x) .
$$

And then we can find the outer solution using the same routine as in the case of ordinary differential equation with integer order derivatives.

To find the inner solution, we also follow the same routine as in the case of differential equation with integer order derivative, with the difference that after changing the variable we make use of Atangana's beta-rule provided in Theorem 5. We will illustrate this with an example.

Example 9. Consider the following fractional singular perturbation problem:

$$
\varepsilon y^{\prime \prime}+(1+\varepsilon){ }_{0}^{A} D_{x}^{\alpha}[y(x)]+y=0, \quad 0<\varepsilon \ll 1,
$$

subject to the following initial and boundary conditions:

$$
y(0)=0, \quad y(1)=1
$$

Outer solution, valid for $t=O(1)$, implying equation (33) is reduced to

$$
{ }_{0}^{A} D_{x}^{\alpha}[y(x)]+y=0 .
$$

But the solution is two times differentiable; this will imply that the fraction version will be rewritten as

$$
\left(x+\frac{1}{\Gamma(\beta)}\right)^{1-\alpha} y^{\prime}+y=0,
$$

by which the solution is given as

$$
y(x, \beta)=c \operatorname{Exp}\left[-\frac{(x+1 / \Gamma(\alpha))^{2-\alpha}}{2-\alpha}\right] .
$$

Applying the condition $y(0)=0$, we have $c=0$; applying the boundary condition $y(1)=1$, we have $c=\operatorname{Exp}\left[(1 / \Gamma(\beta))^{2-\alpha} /\right.$ $(2-\alpha)]$. This is not possible; therefore, according to the matched asymptotic method, we consider the outer solution $c=\operatorname{Exp}\left[(1 / \Gamma(\beta))^{2-\alpha} /(2-\alpha)\right]$. Therefore, the following solution is the leading-order solution in the outer region.

The inner solution is valid for $t=O(\varepsilon)$. In the inner region, $t$ and $\varepsilon$ are both tiny, but of comparable size, so define the new $O(1)$ time variable $\xi=x / \varepsilon$. Rescaling the original fractional boundary value problem by replacing $t$ with $\xi \varepsilon$, the problem becomes

$$
\frac{1}{\varepsilon} y^{\prime \prime}(\xi)+(1+\varepsilon)^{A} D_{x}^{\alpha}[y(\xi \varepsilon)]+y(\xi)=0 .
$$

The fractional part can be converted using first the fact that $y$ is differentiable and secondly using theorem to obtained

$$
\frac{1}{\varepsilon} y^{\prime \prime}(\xi)+\left(\xi+\frac{1}{\Gamma(\alpha)}\right)^{1-\alpha} \frac{1}{\varepsilon}(1+\varepsilon) y^{\prime}(\xi)+y(\xi)=0 .
$$

Of course, by multiplying by the small parameter and taking it to be zero, we will obtain

$$
y^{\prime \prime}(\xi)+\left(\xi+\frac{1}{\Gamma(\alpha)}\right)^{1-\alpha} y^{\prime}(\xi)=0
$$


The solution is given as

$$
\begin{aligned}
& y(\xi, \beta, \varepsilon) \\
& =C \int \operatorname{Exp}\left[-\frac{(\xi+1 / \Gamma(\alpha))^{2-\alpha}}{2-\alpha}\right] d \xi \\
& =C\left(\left(\frac{1}{\Gamma(\alpha)}+\xi\right)\right. \\
& \quad \times \operatorname{ExpIntegral} E\left[1+\frac{1}{-2+\alpha}, \frac{(\xi+1 / \Gamma(\alpha))^{2-\alpha}}{2-\alpha}\right] \\
& \left.\quad \times(-2+\alpha)^{-1}\right)+B,
\end{aligned}
$$

where ExpIntegral $E$ is the exponential integral function defined as

$$
\text { ExpIntegral } E[n, z]=\int_{x}^{\infty} \frac{\exp [-x t]}{t^{n}} d t .
$$

Therefore, the general solution for inner part is given as

$$
\begin{aligned}
& y(\xi, \beta, \varepsilon) \\
&= B+C \\
& \quad \times\left(\left(\frac{1}{\Gamma(\alpha)}+\xi\right)\right. \\
& \quad \times \text { ExpIntegral } E\left[1+\frac{1}{-2+\alpha}, \frac{(\xi+1 / \Gamma(\alpha))^{2-\alpha}}{2-\alpha}\right] \\
&\left.\quad \times(-2+\alpha)^{-1}\right) .
\end{aligned}
$$

To find the constants $B$ and $C$, we apply the boundary conditions. First, for $t=0$, which corresponds to the inner region, we have

$$
\begin{aligned}
B+C( & \frac{1}{\Gamma(\alpha)} \\
& \times \text { ExpIntegral } E\left[1+\frac{1}{-2+\alpha}, \frac{(1 / \Gamma(\alpha))^{2-\alpha}}{2-\alpha}\right] \\
& \left.\times(-2+\alpha)^{-1}\right)=0
\end{aligned}
$$

such that the outer solution becomes

$$
\begin{aligned}
y(\xi, \beta, \varepsilon) & \\
=-C & \left(\frac{1}{\Gamma(\alpha)}\right. \\
& \times \text { ExpIntegral } E\left[1+\frac{1}{-2+\alpha}, \frac{(1 / \Gamma(\alpha))^{2-\alpha}}{2-\alpha}\right] \\
& \left.\times(-2+\alpha)^{-1}\right)
\end{aligned}
$$

$$
\begin{aligned}
& +C\left(\left(\frac{1}{\Gamma(\alpha)}+\xi\right)\right. \\
& \quad \times \text { ExpIntegral } E\left[1+\frac{1}{-2+\alpha}, \frac{(\xi+1 / \Gamma(\alpha))^{2-\alpha}}{2-\alpha}\right] \\
& \left.\quad \times(-2+\alpha)^{-1}\right) .
\end{aligned}
$$

Currently working to find the constant $C$, we employ the harmonising method also called matching method. The principal idea here is that the inner and the outer solutions should harmonise for values of $x$ in an intermediary region, where $\varepsilon \ll t \ll 1$. We demand the outer limit of the inner solution to match the limit of the outer solution, which in mathematical formula implies

$$
\lim _{\xi \rightarrow \infty} y_{I}=\lim _{x \rightarrow 0} y_{O}
$$

which corresponds to

$$
\begin{aligned}
C=-1 & \\
& \times\left(\left(\frac{1}{\Gamma(\alpha)}\right)\right. \\
& \times \text { ExpIntegral } E\left[1+\frac{1}{-2+\alpha}, \frac{(1 / \Gamma(\alpha))^{2-\alpha}}{2-\alpha}\right] \\
& \left.\times(-2+\alpha)^{-1}\right)^{-1} .
\end{aligned}
$$

Composite Solution. To have the final matched or composite solution, which is valid, on the whole domain, one of the commonly used methods is the so-called uniform method. This method consists of an addition of the inner and the outer approximations and subtracting their overlapping value $y_{\text {overlap}}$, which would otherwise be counted twice. The overlapping value is the limit of the inner boundary layer solution and the inner limit of the outer solution. In mathematical formula, we have the following:

$$
y(x)=y_{I}(x)+y_{O}(x)-y_{\text {overlap }}
$$

\section{Conclusion}

Boundary layers problems are a class of problems that can be found in many areas of real world situations. In particular, numerous extraordinary disconcerted differential equations have explanations that transform precipitously in a contracted section. This may happen in a preliminary layer everywhere there is a speedy modification of original discrepancy surroundings to a quasisteady state, in a boundary layer where the solution away from the borderline adjusts to a boundary condition, or in an interior layer such as a propagation wave front. The method in charge of solving these equations is the so-called matched asymptotic method. Due to the nature of this method, it was not possible to use it to solve the fractional boundary layer problems, because the existing 
fractional order derivative does not obey the so-called chain rule. In order to solve this problem, we have presented a relatively novel derivative, which is more suitable than the existing one. The properties of this derivative allowed us to extend the matched asymptotic method to the scope of fractional calculus. We have presented in detail an example to exemplify the method.

\section{Conflict of Interests}

The authors declare that there is no conflict of interests regarding the publication of this paper.

\section{Acknowledgment}

The first author Abdon Atangana would like to thank Claude Leon Foundation for their financial support.

\section{References}

[1] H. Martin, “The generalized Lévêque equation and its practical use for the prediction of heat and mass transfer rates from pressure drop," Chemical Engineering Science, vol. 57, no. 16, pp. 3217-3223, 2002.

[2] J. Kestin and L. N. Persen, "The transfer of heat across a turbulent boundary layer at very high prandtl numbers," International Journal of Heat and Mass Transfer, vol. 5, no. 5, pp. 355-371, 1962.

[3] H. Schlichting, Boundary-Layer Theory, McGraw-Hill, New York, NY, USA, 7th edition, 1979.

[4] A. H. Nayfeh, Perturbation Methods, John Wiley \& Sons, New York, NY, USA, 2000.

[5] F. Verhulst, Methods and Applications of Singular Perturbations: Boundary Layers and Multiple Timescale Dynamics, vol. 50 of Texts in Applied Mathematics, Springer, New York, NY, USA, 2005.

[6] L. E. Fraenkel, "On the method of matched asymptotic expansions Part I. A matching principle," Proceedings of the Cambridge Philosophical Society, vol. 65, pp. 209-231, 1969.

[7] A. Atangana and A. Secer, "A note on fractional order derivatives and table of fractional derivatives of some special functions," Abstract and Applied Analysis, vol. 2013, Article ID 279681, 8 pages, 2013.

[8] M. Caputo, "Linear models of dissipation whose Q is almost frequency independent, part II," Geophysical Journal International, vol. 13, no. 5, pp. 529-539, 1967.

[9] G. Jumarie, "Modified Riemann-Liouville derivative and fractional Taylor series of nondifferentiable functions further results," Computers and Mathematics with Applications, vol. 51, no. 9-10, pp. 1367-1376, 2006.

[10] D. Baleanu, J. A. Tenreiro Machado, C. Cattani, M. C. Baleanu, and X.-J. Yang, "Local fractional variational iteration and decomposition methods for wave equation on Cantor sets within local fractional operators," Abstract and Applied Analysis, vol. 2014, Article ID 535048, 6 pages, 2014.

[11] R. Khalil, M. Al Horani, A. Yousef, and M. Sababheh, "A new definition of fractional derivative," Journal of Computational and Applied Mathematics, vol. 264, pp. 65-70, 2014.

[12] M. Abu Hammad and R. Khalil, "Conformable fractional Heat differential equation," International Journal of Pure and Applied Mathematics, vol. 94, no. 2, pp. 215-221, 2014. 


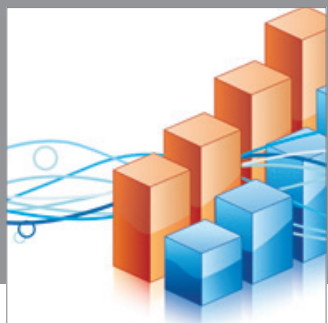

Advances in

Operations Research

mansans

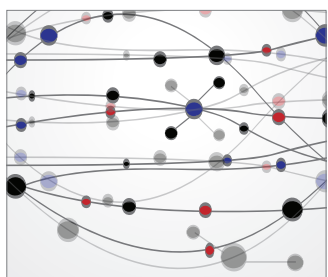

The Scientific World Journal
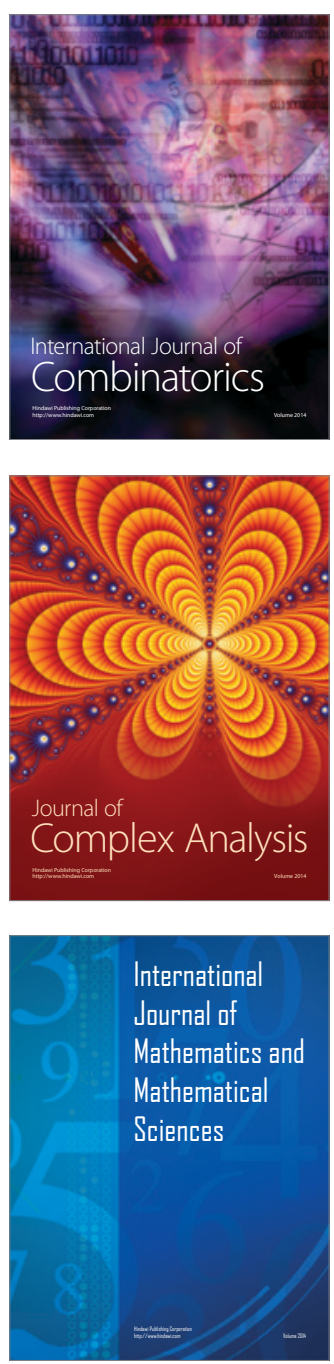
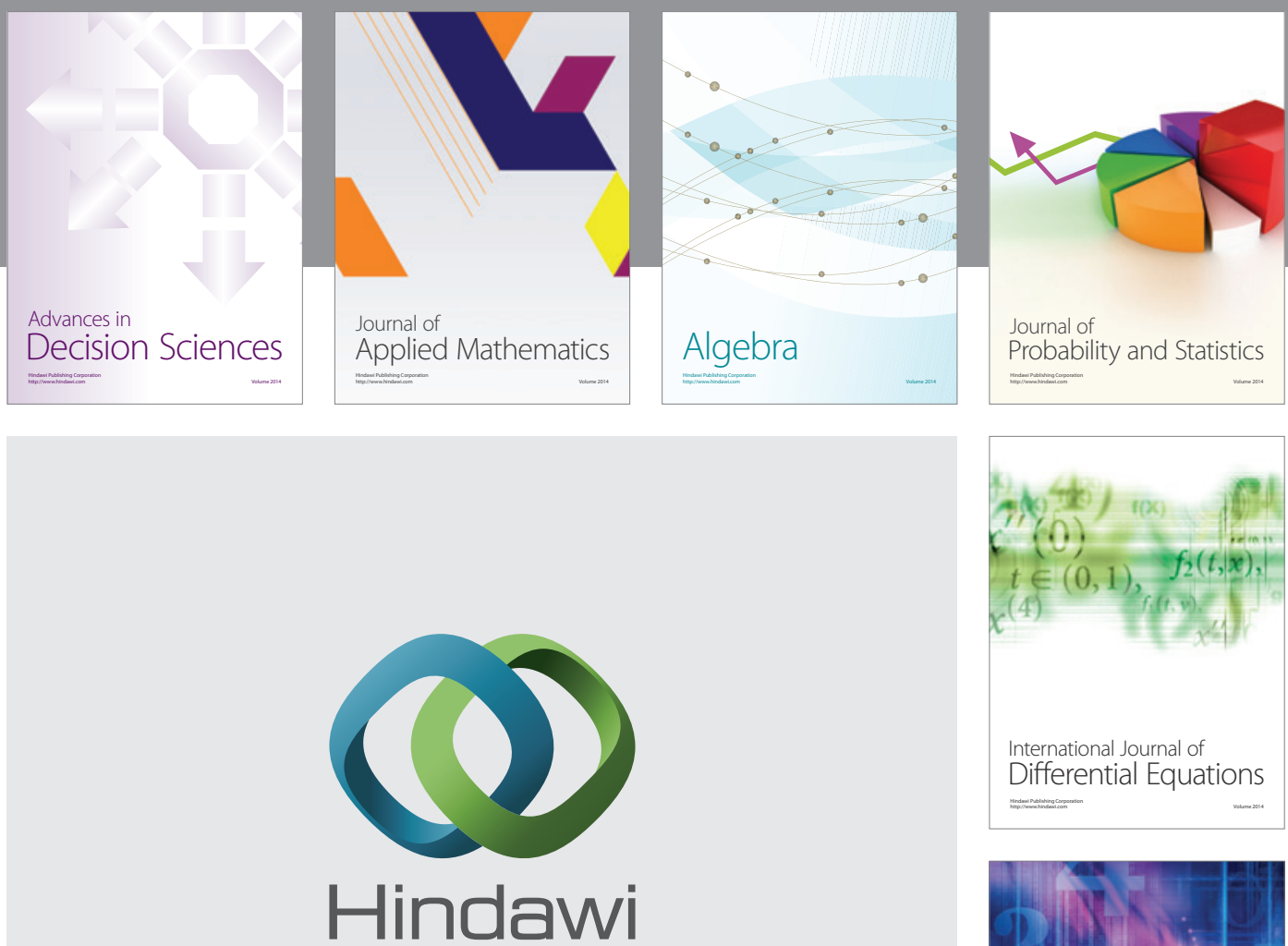

Submit your manuscripts at http://www.hindawi.com
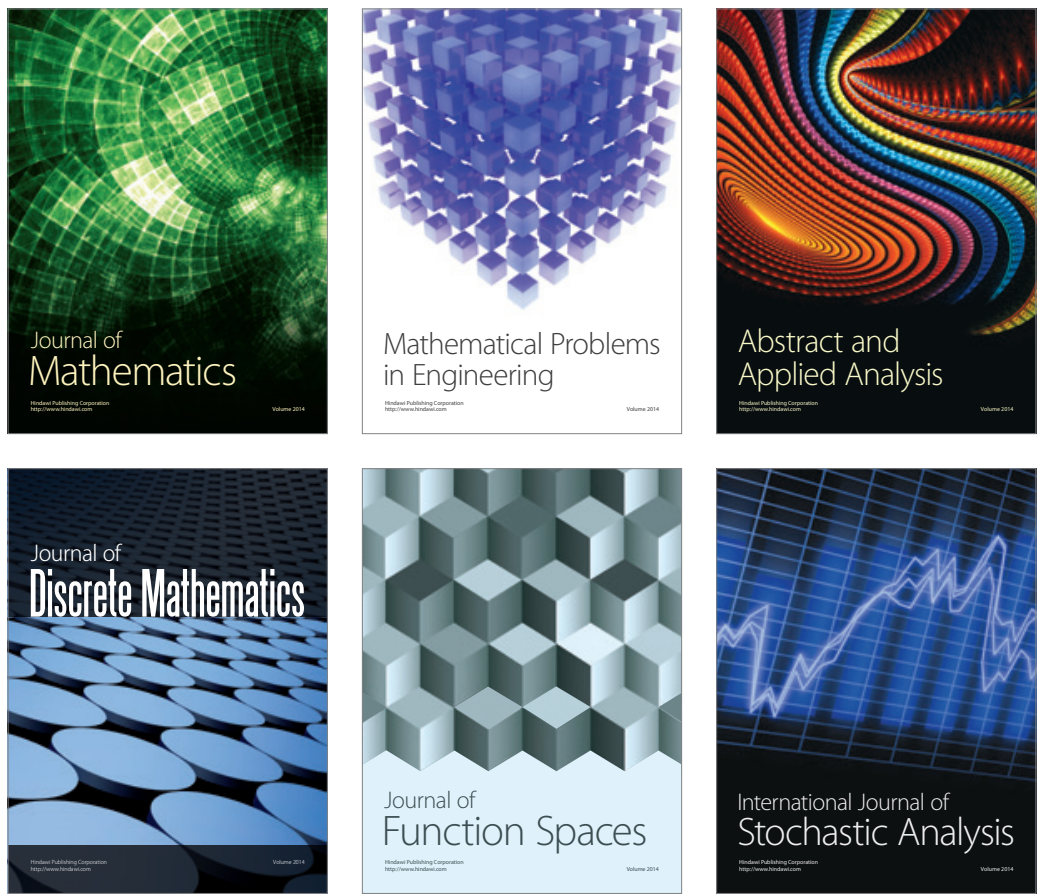

Journal of

Function Spaces

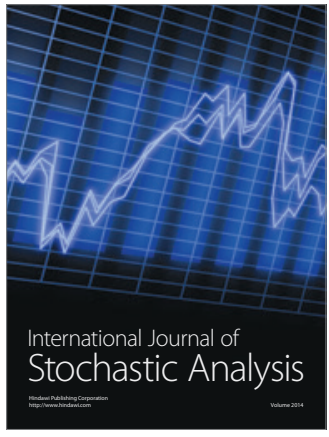

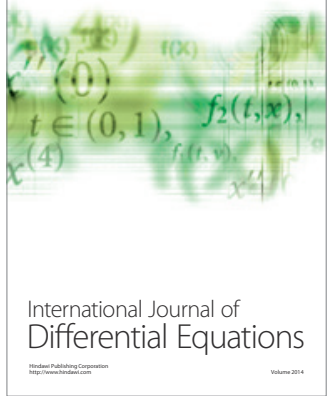
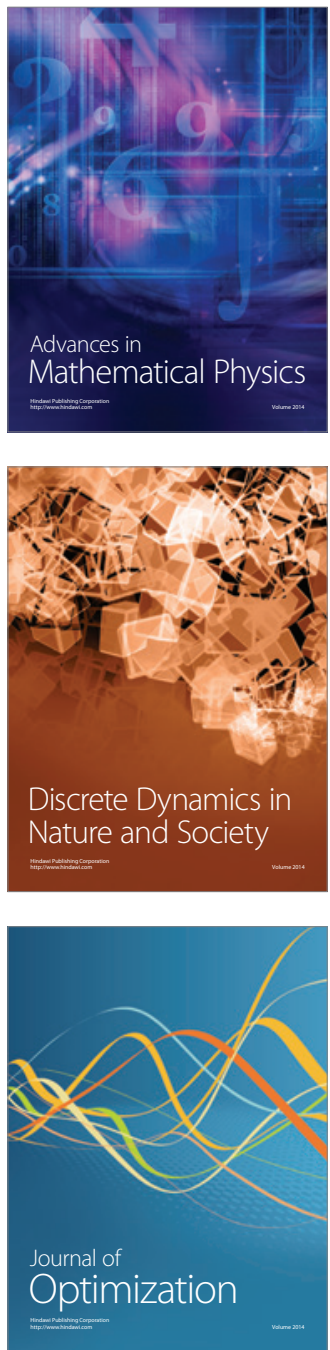